\title{
Hybridizing Multi-pass and Multi-plate Bulk Compression
}

\author{
M. Seidel, ${ }^{1}$ P. Balla, ${ }^{1}$ T. Binhammer, ${ }^{2}$ M. Frede, ${ }^{2}$ G. Arisholm, ${ }^{3}$ L. Winkelmann, ${ }^{1}$ I. Hartl, ${ }^{1}$ and C.M. Heyl ${ }^{1,4}$ \\ 1. Deutsches Elektronen-Synchrotron DESY, Notkestraße 85, 22607 Hamburg, Germany \\ 2. neoLASE, Hollerithallee 17, 30419 Hannover, Germany \\ 3. FFI (Norwegian Defence Research Establishment), P. O. Box 25, NO-2027 Kjeller, Norway \\ 4. Helmholtz-Institute Jena, Fröbelstieg 3, 07743 Jena, Germany
}

With the advent of ultrafast $\mathrm{Yb}$-ion based disk, slab and fibre lasers, nonlinear pulse compression methods became indispensable for the generation of high average power sub-100 fs pulses. In particular, spectral broadening in Herriott-type multi-pass cells (MPC) [1] has been established as a novel tool for pulse compression in the $5-100 \mathrm{MW}$ peak power range where neither solid-core fibre nor hollow-core capillaries work efficiently. The operation in the critical self-focusing regime becomes feasible through repetitive refocusing of the cell mirrors. Analogously, the multi-plate approach [2], which has mainly been used for broadband continuum generation, relies on the nonlinear refocusing of the Kerr media [3]. By introducing the hybridization of both methods, we demonstrate compression factors up to 14 . To the best of our knowledge this exceeds all single-stage compression factors achieved with bulk-based spectral broadening to date (Fig. 1e).

In our experiments, we used a home-built fibre-laser front-end and a neoLASE Yb:YAG amplifier [4]. The source delivered $10 \mathrm{~Hz}$ bursts of up to 800 pulses with $100 \mu \mathrm{J}$ energy, 880 fs duration and $1 \mathrm{MHz}$ repetition rate. Our MPC consisted of dielectric focusing mirrors with $200 \mathrm{~mm}$ radius of curvature. Inserting a single $6 \mathrm{~mm}$ thick silica plate resulted in broadening to a Fourier limit of about $100 \mathrm{fs}$ and the characteristic phase jump in the retrieved FROG trace known from bulk compression [3]. However, when using three $1 \mathrm{~mm}$ thick plates with about $3 \mathrm{~cm}$ mutual distance, the phase jump was not observed anymore and clean pulse compression was achieved (Fig. $1 \mathrm{~b}$ ). With a $380 \mathrm{~mm}$ long cell and $85 \mu \mathrm{J}$ pulse energy, we compressed the pulses to $65 \mathrm{fs}$ by means of chirped mirrors (Fig. $1 \mathrm{c}$ ). The full laser output was sent through a $350 \mathrm{~mm}$ long MPC with $85 \%$ transmission. The pulses were compressed to $75 \mathrm{fs}$ with a grating pair. The $\mathrm{M}^{2}$ factor was $<1.4$ and the spectrum was stable over a measurement period of 15 hours. A further increase of input energy and broadening factor for the $380 \mathrm{~mm}$ cell is possible if the mode-matching of the input beam is adjusted to the nonlinear self-lensing of the Kerr media. Simulations with the SISYFOS package [3] show that, contrary to linear (or low power) modematching, the peak intensity in the nonlinear media is kept well below the damage threshold. Moreover, compression factors exceeding 20 are predicted if 6 instead of 3 plates are placed into the MPC. Furthermore, the nonlinear effects become saturated, yielding an additional increase of the output spectrum stability (Fig. 1d).

In conclusion, we have shown that hybridizing multi-plate and multi-pass bulk broadening techniques results in a compact pulse compression setup capable of generating clean, nearly Fourier-limited sub-100 fs pulses. We have experimentally demonstrated compression factors of more than 10 and simulated factors of more than 20 . These are expected to be experimentally confirmed soon. With that, the simple-to-implement and robust bulk broadening method becomes also increasingly competitive to gas-filled MPCs [5, 6].

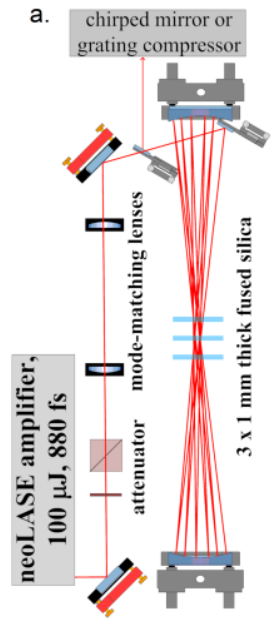

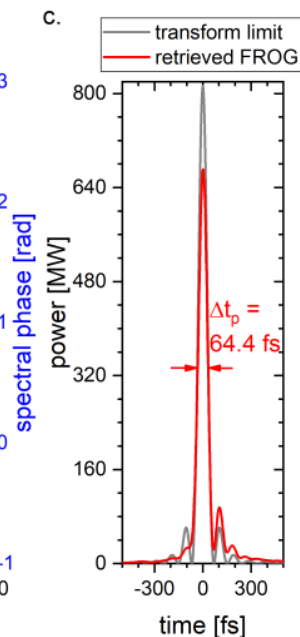
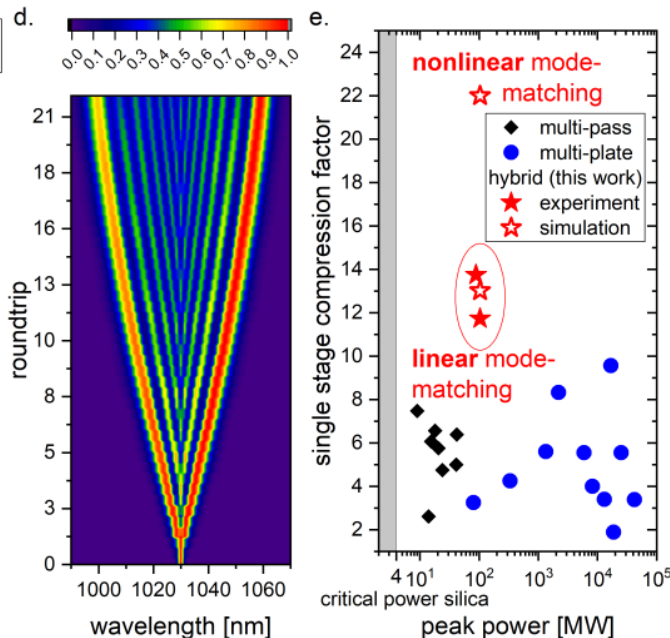

Fig. 1 a. Experimental setup. b. Retrieved FROG spectrum in comparison to grating spectrometer spectrum after 23 roundtrips in the $380 \mathrm{~mm}$ cavity. c. Corresponding retrieved pulse in comparison to the Fourier transformed spectrum. d. Simulation of spectral broadening with 6 plates in the MPC and nonlinear mode-matching. e. Reported single stage compression factors of bulk multi-pass and multi-plate experiments in comparison to the results presented here. Encircled are the experimental $75 \mathrm{fs}$ and $65 \mathrm{fs}$ compression results (solid red stars) and the benchmarking simulation (hollow star). The hollow star at the top predicts 40 fs pulses for 6 plates if mode-matching accounts for self-focusing.

\section{References}

[1] J. Schulte et al., Opt. Lett. 41, 4511 (2016).

[2] C.-H. Lu et al., Optica 1, 400 (2014).

[3] M. Seidel et al., Opt. Exp. 24, 9412 (2016).

[4] O. Akcaalan et al., submitted to Europhoton 2020

[5] M. Kaumanns et al., Opt. Lett 43, 5877 (2018).

[6] P. Balla et al., Opt. Lett. 45, 2572 (2020). 